\title{
COMBINATORY RECURSIVE OBJECTS OF ALL FINITE TYPES
}

BY HASKELL B. CURRY

Communicated by L. Henkin, June 10, 1964

In a recent paper [1] Grzegorczyk has shown that several concepts of recursive functional of finite type can be represented in a system $R$ which is closely related to combinatory logic. The exact nature of that relationship is not pointed out there, and the author appears to be unaware of it. The purpose of this note is to record some facts about this relationship; in so doing I shall dispose of an "open problem" which the author proposes in his footnote $3, \mathrm{p} .74$. The topic is a part of an investigation now under way in preparation for the second volume of [2]. ${ }^{1}$ The notation used is that of [2]. Thus $\mathbf{F} \alpha \beta$ is Grzegorczyk's $(\alpha \beta) ; X Y$ is his $(X, Y)$; and $\mathbf{W}$ is his $\mathbf{D}$.

In combinatory logic, as developed previous to [2], the natural numbers were represented, following Church [3], by certain combinators $\mathbf{Z}_{n}$ called iterators. As stated in [2, pp. $174 \mathrm{ff}$.], a considerable arithmetic can be developed on this basis. This development is not contained in [2] for the reasons stated there on p. 7; some parts of it are, however, expounded in $\S \S 5-6$ of [4] and Chapter IV of [5]. The basic ideas are due primarily to Kleene, Rosser, and Bernays. On this basis there are given in [4] two definitions of a combinator $\mathbf{R}$, called the primitive recursion combinator, such that, for any obs $a, b$,

$$
\mathbf{R} a b \mathbf{Z}_{0}=a, \quad \mathbf{R} a b \mathbf{Z}_{n+\mathbf{1}}=b \mathbf{Z}_{n}\left(\mathbf{R} a b \mathbf{Z}_{n}\right) .
$$

Furthermore, one can show, by natural induction on $n$, that for any $\alpha$

$$
\vdash \mathbf{F}(\mathbf{F} \alpha \alpha)(\mathbf{F} \alpha \alpha) \mathbf{Z}_{n} \text {. }
$$

This motivates the assumption (here $\mathbf{N}$ is the category of natural numbers)

$$
\vdash \mathbf{F N}\left(\mathbf{F}\left(\mathbf{F}_{\alpha \alpha)}(\mathbf{F} \alpha \alpha)\right)\right. \text {. }
$$

Furthermore, one can show that for any $\alpha$

$$
\vdash \mathbf{F}_{2} \alpha\left(\mathbf{F}_{2} \mathbf{N} \alpha \alpha\right)(\mathbf{F} \mathbf{N} \alpha) \mathbf{R} \text {. }
$$

Although the basic theory of functionality was not in existence at

${ }^{1}$ Supported in part by National Science Foundation grant number GP 1763. 
that time, the arguments establishing the foregoing can be formalized therein, provided one uses the second definition for $\mathbf{R}$ in [4], viz., that in $\$ 5.6$.

In [1] the numbers are postulated as independent entities. Some correspondence with Dana Scott in 1963, in which he proposed a different representation of the integers, has also suggested the same idea. If angle brackets are used to indicate the formal analogues of the arithmetical notions symbolized between them, this means that $\langle 0\rangle$ and $\langle\sigma\rangle$ (where $\sigma$ is the successor function) are taken as atoms, with the new axioms

$$
\vdash \mathbf{N}\langle 0\rangle, \quad \vdash \mathbf{F N} \mathbf{N}\langle\sigma\rangle .
$$

Now suppose that we postulate also a $\mathbf{Z}$ such that

$$
\begin{gathered}
\mathbf{Z}\langle n\rangle=\mathbf{Z}_{n}, \\
1 \mathbf{F} \mathbf{N}(\mathbf{F}(\mathbf{F} \alpha \alpha)(\mathbf{F} \alpha \alpha)) \mathbf{Z} .
\end{gathered}
$$

Then the arguments of the preceding paragraph can all be carried out. One simply replaces all numbers $\mathbf{Z}_{n}$ by $\mathbf{Z}\langle n\rangle$ or $\langle n\rangle$ according to the context (with corresponding adjustments for numerical variables), and uses ( 7 ) instead of (3). Thus, in the system $\Omega^{\prime}$ so constituted one can derive (1) and (4) (with $\langle n\rangle$ for $\mathbf{Z}_{n}$ ), etc., by the basic theory of functionality.

Let $\Omega^{\prime \prime}$ be the system formed by postulating $\mathbf{R}$ and (1), (4) (as modified) instead of (6), (7). Then the $\mathbf{Z}$ defined by

$$
\mathbf{Z} \equiv \mathbf{R}(\mathbf{K I})(\mathbf{K}(\mathbf{S B}))
$$

will satisfy (6) and (7). Thus $\mathcal{R}^{\prime \prime}$ is equivalent to $R^{\prime}$.

In Grzegorczyk's $R$, the combinators $\mathbf{I}, \mathbf{K}, \mathbf{C}, \mathbf{B}, \mathbf{W}$, and $\mathbf{R}$ are postulated with type indications which are precisely the functional characters assigned to these combinators in the basic theory of functionality. If we drop these type indexes, the functional characters being assigned by the axioms, we have a formulation of $R^{\prime \prime}$. Then $R$ and $\Omega^{\prime \prime}$ are equivalent. Clearly $R \subset \mathcal{R}^{\prime \prime}$. If $X$ is in $R^{\prime \prime}$, then by theorems of $[2, \S 9 \mathrm{C}]$ (with a modified equality), there is a $\xi$ such that there is an F-deduction of $\vdash \xi X$. By the Subject-construction Theorem $[2, \S 9 \mathrm{~B} 1]$, there is a unique axiom corresponding to every instance of a combinator; if all these instances are indexed with the functional characters so determined, the argument will be valid in $\Omega$.

Thus the systems $R, \mathbb{R}^{\prime}, \mathbb{R}^{\prime \prime}$ are all equivalent. Since $\mathbf{Z}$ can also be defined as $[x, y, z] \mathbf{R}^{\prime} z x y$, where $\mathbf{R}^{\prime}$ is defined as in [1], this disposes of Grzegorczyk's open problem. 
The foregoing argument uses (in [4]) pairing combinators $\mathbf{D}, \mathbf{D}_{\mathbf{1}}$, $D_{2}$ such that

$$
\mathbf{D}_{1}(\mathbf{D} x y)=x, \quad \mathbf{D}_{2}(\mathbf{D} x y)=y .
$$

The $D_{2}$ of [4] is one such $\mathbf{D}$; it has the property that for any $\alpha$ there is a $\delta$ such that

$$
\vdash F_{2} \alpha \alpha \delta \mathbf{D}, \quad-F \delta \alpha D_{1}, \quad-F \delta \alpha D_{2} .
$$

All we need to know concerning $\mathbf{D}$, in order to carry out the foregoing, is that (7) and (9) hold for $\alpha \equiv \mathbf{N}$. There are several possible D's (with associated $\mathbf{D}_{1}, \mathbf{D}_{2}$ ) which have these properties. But any such D is only suitable for forming homogeneous pairs, i.e., those where the two constituents are of the same type.

In $\S 3$ of [1] there is a method for defining pairing functions for certain nonhomogeneous cases. This argument can be generalized as follows. Let (8), (9) hold, and let

$$
\begin{gathered}
\xi \equiv \mathbf{F}_{m} \beta_{1} \beta_{2} \cdots \beta_{m} \alpha, \quad \eta \equiv \mathbf{F}_{n} \gamma_{1} \cdots \gamma_{n} \alpha \\
\zeta \equiv \mathbf{F}_{m+n} \beta_{1} \cdots \beta_{m} \gamma_{1} \cdots \gamma_{n} \delta .
\end{gathered}
$$

Let $b_{1}, \cdots, b_{m}, c_{1}, \cdots, c_{n}$ be constants such that

$$
\vdash \beta_{i} b_{i}, \quad \vdash \gamma_{j} c_{j} .
$$

Then define

$$
\begin{aligned}
\mathbf{D}^{\xi \eta} & \equiv\left[x, y, u_{1}, \cdots, u_{m}, v_{1}, \cdots, v_{n}\right] \mathbf{D}\left(x u_{1} \cdots u_{m}\right)\left(y v_{1} \cdots v_{n}\right), \\
\mathbf{D}_{1}^{\xi \eta} & \equiv\left[z, u_{1}, \cdots, u_{m}\right] \mathbf{D}_{1}\left(z u_{1} \cdots u_{m} c_{1} \cdots c_{n}\right), \\
\mathbf{D}_{2}^{\xi \eta} & \equiv\left[z, v_{1}, \cdots, v_{n}\right] \mathbf{D}_{2}\left(z b_{1} \cdots b_{m} v_{1} \cdots v_{n}\right) .
\end{aligned}
$$

Then we have

$$
\begin{gathered}
\mathbf{D}_{1}^{\xi \eta}\left(\mathbf{D}^{\xi \eta} x y\right)=x, \quad \mathbf{D}_{2}^{\xi \eta}\left(\mathbf{D}^{\xi \eta} x y\right)=y, \\
\vdash \mathbf{F}_{2} \xi \eta \xi \mathbf{D}^{\xi \eta}, \quad \vdash \mathbf{F} \xi \xi \mathbf{D}_{1}^{\xi \eta}, \quad \vdash \mathbf{F} \xi \eta \mathbf{D}_{2}^{\xi \eta} .
\end{gathered}
$$

What particular definition of $\mathbf{D}, \mathbf{D}_{1}, \mathbf{D}_{2}$ is used, whether or not the type indices are numbers, and whether or not $\alpha$ is $\mathbf{N}$-these are all irrelevant. For the cases considered in [1] the constants (10) can be of the form $\mathbf{K}^{k}\langle 0\rangle$. 


\section{BIBLIOGRAPHY}

1. A. Grzegorczyk, Recursive functionals in all finite types, Fund. Math. 54 (1964), 73-93.

2. H. B. Curry and R. Feys, Combinatory logic, Vol. I, Amsterdam, 1958.

3. A. Church, $A$ set of postulates for the foundation of logic (Second paper), Ann. of Math. (2) 34 (1933), 839-864.

4. H. B. Curry, The paradox of Kleene and Rosser, Trans. Amer. Math. Soc. 50 (1941), 454-516.

5. Bruce Lercher, Strong reduction and recursion in combinatory logic, Thesis, The Pennsylvania State University, University Park, Pa., 1963.

The Pennsylvania State University 